\title{
First-Principles Definition and Measurement of Planetary Electromagnetic-Energy Budget
}

\author{
Michael I. Mishchenko \\ NASA Goddard Institute for Space Studies \\ James A. Lock \\ Cleveland State University, j.lock@csuohio.edu \\ Andrew A. Lacis \\ NASA Goddard Institute for Space Studies \\ Larry D. Travis \\ NASA Goddard Institute for Space Studies \\ Brian Cairns \\ NASA Goddard Institute for Space Studies \\ Follow this and additional works at: https://engagedscholarship.csuohio.edu/sciphysics_facpub \\ Part of the Physics Commons \\ How does access to this work benefit you? Let us know!
}

\section{Original Citation}

Michael I. Mishchenko et al., "First-principles definition and measurement of planetary electromagneticenergy budget," J.Opt.Soc.Am.A 33 (6), 1126-1132 (2016).

\section{Repository Citation}

Mishchenko, Michael I.; Lock, James A.; Lacis, Andrew A.; Travis, Larry D.; and Cairns, Brian, "First-Principles Definition and Measurement of Planetary Electromagnetic-Energy Budget" (2016). Physics Faculty Publications.

388.

https://engagedscholarship.csuohio.edu/sciphysics_facpub/388

This Article is brought to you for free and open access by the Physics Department at EngagedScholarship@CSU. It has been accepted for inclusion in Physics Faculty Publications by an authorized administrator of EngagedScholarship@CSU. For more information, please contact library.es@csuohio.edu. 


\title{
First-principles definition and measurement of planetary electromagnetic-energy budget
}

\author{
Michael I. Mishchenko, * James A. Lock, Andrew A. Lacis, Larry D. Travis, and Brian Cairns
}

\begin{abstract}
The imperative to quantify the Earth's electromagnetic-energy budget with an extremely high accuracy has been widely recognized but has never been formulated in the framework of fundamental physics. In this paper we give a first-principles definition of the planetary electromagnetic-energy budget using the Poynting-vector formalism and discuss how it can, in principle, be measured. Our derivation is based on an absolute minimum of theoretical assumptions, is free of outdated notions of phenomenological radiometry, and naturally leads to the conceptual formulation of an instrument called the double hemispherical cavity radiometer (DHCR). The practical measurement of the planetary energy budget would require flying a constellation of several dozen planet-orbiting satellites hosting identical well-calibrated DHCRs. (c) 2016 Optical Society of America
\end{abstract}

OCIS codes: (010.5630) Radiometry; (010.0280) Remote sensing and sensors; (010.5620) Radiative transfer; (010.3920) Meteorology.

\section{INTRODUCTION}

The global climate of a planet (or an exoplanet) is ultimately defined by the planet's electromagnetic-energy budget, i.e., by the difference between the incoming electromagnetic energy from the star and the outgoing electromagnetic energy scattered and emitted by the planet [1-13]. The imperative to monitor and model the Earth's electromagnetic-energy budget and to do that with an extremely high accuracy $(\sim 0.1 \%$ or even better) has been articulated in many recent publications (see, e.g., [14-29] and references therein). Yet, despite the supreme importance of this problem, it has never been formulated in the framework of first-principles physics. Instead, obsolete notions of phenomenological radiometry [30-38] have exclusively been used, even though many of them had predated the discovery of the electromagnetic nature of light [39]. Among the typical misconceptions have been the generally wrong belief that matter interacts with radiant energy rather than with the electromagnetic field; the misapprehension that what propagates in space is electromagnetic energy rather than electromagnetic waves; the heuristic notions of radiance and multidirectional radiation fluxes; the misuse of electromagnetic radiation as consisting of "incoherent pencils of rays" or streams of localized point-like particles of light called "photons"; etc.

The outdated and deceptive character of these and similar misconceptions has by now been thoroughly exposed (see, e.g., [40-46] and numerous references therein). This makes it quite imperative to reformulate the planetary energy-budget problem in terms of a fundamental electromagnetic theory. Indeed, the highly demanding accuracy requirement quoted above appears to be utterly incommensurate with the traditional heuristic way of addressing this problem. Hence our main objective is to give a physics-based definition of the planetary electromagnetic-energy budget and discuss how it can, in principle, be measured. We intentionally focus on presenting a formalism that is based on an absolute minimum of theoretical assumptions rather than on a specific discussion of the technical feasibility of the requisite measurement approach.

\section{GENERAL FRAMEWORK}

Although the most fundamental theory of field-matter interactions available today is quantum electrodynamics (QED) $[43,47,48]$, it is clear that technical complexities of using the QED to describe electromagnetic interactions of the enormous number of elementary particles constituting a star and a planet are overwhelming and not especially instructive. Fortunately, the explicit use of the QED can be avoided by recognizing that once an elementary source has scattered or emitted electromagnetic radiation, the resulting field at a large distance from the source (i.e., in its far zone) can be considered an outgoing spherical electromagnetic wave. As a consequence, the instantaneous electromagnetic field at a point in a vacuum surrounding the planet is a superposition of a vast number of spherical electromagnetic waves centered at the various elementary sources involved. This factor allows for the use of the 

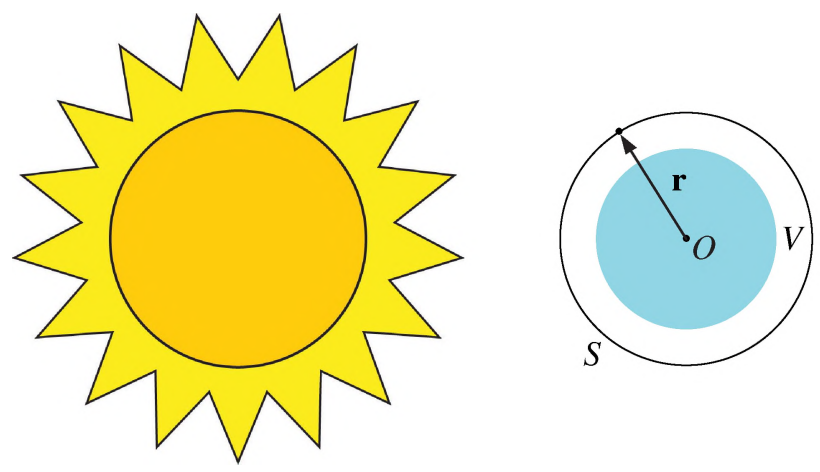

Fig. 1. Planetary climate is defined by difference between incoming and outgoing electromagnetic energy.

semiclassical approach wherein only matter is quantized, while the electromagnetic field is treated classically [43,49-51].

Let us surround the planet by a concentric sphere $S$ with a radius $R_{S}$ exceeding the radius of the planet (Fig. 1), denote by $V$ the volume bounded by $S$, and assume that the planet is an electrically neutral configuration of elementary charges. Then, according to the Poynting theorem (see $\$ 31$ of [52] or Section 2.3 of [53]), the time-averaged electromagnetic-energy budget of the planet is defined by the following integral,

$$
I_{\mathrm{p}}=-\int_{S} \mathrm{~d}^{2} \mathbf{r}\langle\mathbf{S}(\mathbf{r}, t)\rangle \cdot \hat{\mathbf{r}}[\mathrm{W}],
$$

where $t$ is time, $\mathbf{r}$ is the position vector originating at the center of the planet $O, \hat{\mathbf{r}}=\mathbf{r} / R_{S}$ is the unit vector in the direction of the local outward normal to $S,\langle\cdots\rangle$ denotes averaging over a sufficiently long period of time (e.g., the time interval necessary to take a measurement), and $\mathbf{S}(\mathbf{r}, t)$ is the instantaneous local Poynting vector. The latter is given by the vector product of the electric, $\mathbf{E}$, and magnetic, $\mathbf{H}$, field vectors,

$$
\begin{aligned}
\mathbf{S}(\mathbf{r}, t) & =\mathbf{E}(\mathbf{r}, t) \times \mathbf{H}(\mathbf{r}, t) \\
& =\operatorname{Re}[\tilde{\mathbf{E}}(\mathbf{r}, t)] \times \operatorname{Re}[\tilde{\mathbf{H}}(\mathbf{r}, t)]\left[\mathrm{Wm}^{-2}\right],
\end{aligned}
$$

where $\tilde{\mathbf{E}}(\mathbf{r}, t)$ is the complex-valued electric field vector, $\tilde{\mathbf{H}}(\mathbf{r}, t)$ is the complex-valued magnetic field vector, and "Re" stands for "the real part of." The total electric and magnetic fields at any point $\mathbf{r} \in S$ are superpositions of those contributed by the star (subscript "s") and the planet (subscript "p"):

$$
\begin{aligned}
\tilde{\mathbf{E}}(\mathbf{r}, t) & =\tilde{\mathbf{E}}_{s}(\mathbf{r}, t)+\tilde{\mathbf{E}}_{\mathrm{p}}(\mathbf{r}, t), \\
\tilde{\mathbf{H}}(\mathbf{r}, t) & =\tilde{\mathbf{H}}_{\mathrm{s}}(\mathbf{r}, t)+\tilde{\mathbf{H}}_{\mathrm{p}}(\mathbf{r}, t) .
\end{aligned}
$$

If $I_{\mathrm{p}}>0$ then electromagnetic energy is accumulated and dissipated inside $V$, which obviously causes a warming effect. If $I_{\mathrm{p}}<0$ then the planet is losing electromagnetic energy and hence is cooling. If $I_{\mathrm{p}}=0$ then the planet is in the state of precise electromagnetic-energy balance.

In the framework of the semiclassical approach based on microscopic electromagnetics, the Poynting vector is the only quantity characterizing the transport of electromagnetic energy. The fact that $\mathbf{S}(\mathbf{r}, t)$ is a monodirectional entity completely rules out physical coexistence of multiple energy fluxes at an observation point $\mathbf{r}$. This, of course, is in a stark contrast to one of the main premises of the phenomenological radiative transfer theory [36-38]. Furthermore, the derivation of the Poynting theorem (i.e., electromagnetic energy conservation) $[54,55]$ says that $\mathbf{S}(\mathbf{r}, t)$ of itself does not have a physical interpretation. Only the integral of the scalar product $\mathbf{S}(\mathbf{r}, t) \cdot \hat{\mathbf{r}}$ over a closed surface is physically meaningful and describes the electromagnetic-energy budget of the entire inscribed volume [56].

Note that coexistence of multiple electromagnetic energy fluxes at a point $\mathbf{r}$ in space also cannot be justified by invoking the notion of "streams of photons flowing through $\mathbf{r}$ in different directions." Indeed, the real QED photons have no position operator and no wave function in the coordinate representation. As such, each QED photon occupies the entire quantization domain rather than being an infinitesimal particle of light localized at a point in space $[40-43,47,48,50,57,58]$. The phenomenological notion of a "Monte Carlo photon" frequently invoked to interpret verbally the Monte Carlo solution of the integral form of the radiative transfer equation [59-66] has nothing to do with physical reality and in no way represents the actual QED photons.

Thus the notion of multidirectional flow of electromagnetic energy through a point in space is generally irrelevant. One can, of course, define directional radiation fluxes purely mathematically as projections of the time-averaged Poynting vector on specific directions. However, this would not change two fundamental facts, i.e., (i) that the Poynting vector is a monodirectional quantity, and (ii) that in and of itself the Poynting vector does not characterize the local flow of electromagnetic energy.

The generality of the Poynting theorem enables us to discuss the planetary energy-budget problem without addressing the enormously complex problem of light-matter interactions at the microphysical level. In what follows we will discuss the main implications of Eqs. (1)-(4) from the perspective of satellite observations and theoretical modeling.

\section{BASIC PROBLEM}

Let us assume that the distance between the star and the planet can be considered essentially infinite. Then in the vicinity of the planet all the spherical waves generated by the elementary charges of the star can be thought of as forming a superposition of polychromatic plane waves with quasi-monochromatic components propagating in directions $\hat{\mathbf{s}}$ within the small solid angle $\Omega_{\mathrm{s}}$ subtended by the star as viewed from the planet (Fig. 2). Obviously, this solid angle is centered around the straight line connecting the centers of the star and the planet (Fig. 1). The frequency range and the spectral distribution of the electromagnetic radiation generated by the star are functions of many parameters [67]. The physical causes of quasi-monochromaticity of the stellar light are discussed in $[68,69]$. We thus have

$$
\begin{aligned}
& \tilde{\mathbf{E}}_{\mathrm{s}}(\mathbf{r}, t)=\int_{\Omega_{s}} \mathrm{~d} \hat{\mathbf{s}} \int_{0}^{\infty} \mathrm{d} \omega \exp (\mathrm{i} k \hat{\mathbf{s}} \cdot \mathbf{r}-\mathrm{i} \omega t) \mathbf{E}_{s}(\hat{\mathbf{s}}, \omega, t), \\
& \tilde{\mathbf{H}}_{s}(\mathbf{r}, t)=\int_{\Omega_{s}} \mathrm{~d} \hat{\mathbf{s}} \int_{0}^{\infty} \mathrm{d} \omega \exp (\mathrm{i} k \hat{\mathbf{s}} \cdot \mathbf{r}-\mathrm{i} \omega t) \mathbf{H}_{s}(\hat{\mathbf{s}}, \omega, t),
\end{aligned}
$$




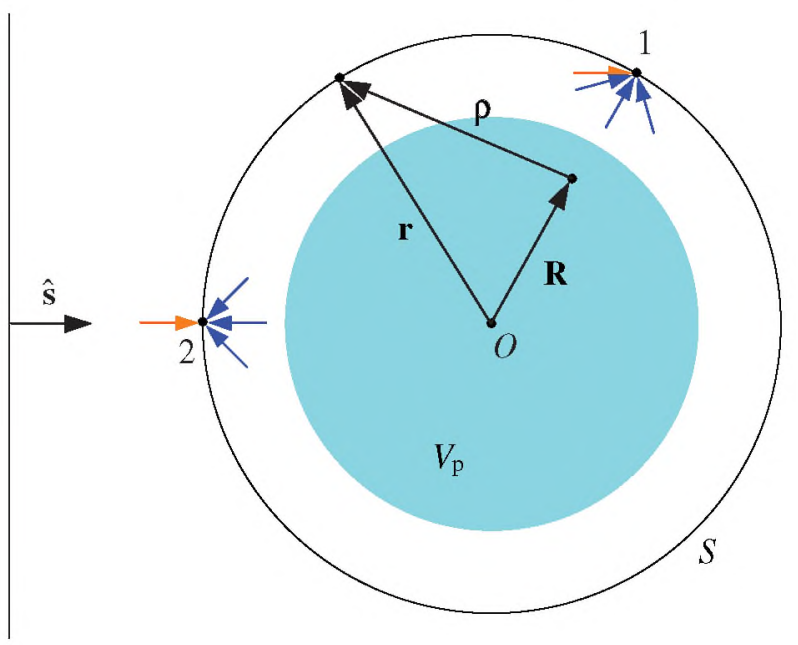

Fig. 2. Illumination geometry.

where $\mathrm{i}=\sqrt{-1} ; \omega$ is the angular frequency; $\epsilon_{0}$ and $\mu_{0}$ are the electric permittivity and the magnetic permeability of a vacuum, respectively;

$$
k=\omega \sqrt{\epsilon_{0} \mu_{0}}
$$

is the wavenumber; $\mathbf{E}_{s}(\hat{\mathbf{s}}, \omega, t)$ is the complex electric-field amplitude of a quasi-monochromatic plane wave generated by the star at the frequency $\omega$ in the direction $\hat{\mathbf{s}} \in \Omega_{\mathrm{s}}$; and

$$
\mathbf{H}_{s}(\hat{\mathbf{s}}, \omega, t)=\sqrt{\frac{\epsilon_{0}}{\mu_{0}}} \hat{\mathbf{s}} \times \mathbf{E}_{\mathrm{s}}(\hat{\mathbf{s}}, \omega, t) .
$$

Note that $\mathbf{E}_{s}(\hat{\mathbf{s}}, \omega, t)$ varies in time much more slowly than the complex-exponential factor $\exp (-\mathrm{i} \omega t)$. The quasi-random temporal fluctuations of the amplitude $\mathbf{E}_{s}(\hat{\mathbf{s}}, \omega, t)$ imply fluctuations of both the amplitude and the phase of the corresponding real electric field vector. In what follows, we will assume that $\mathbf{E}_{s}(\hat{\mathbf{s}}, \omega, t)$ is unaffected by the presence of the planet.

The electromagnetic field contributed by the planet is a superposition of spherical quasi-monochromatic waves centered at various infinitesimal volumes constituting the planetary volume $V_{\mathrm{p}}$ (Fig. 2),

$$
\begin{aligned}
& \tilde{\mathbf{E}}_{\mathrm{p}}(\mathbf{r}, t)=\int_{V_{\mathrm{p}}} \mathrm{d}^{3} \mathbf{R} \int_{0}^{\infty} \mathrm{d} \omega \frac{\exp (\mathrm{i} k \rho-\mathrm{i} \omega t)}{\rho} \mathbf{E}_{\mathrm{p}}(\mathbf{R}, \hat{\boldsymbol{\rho}}, \omega, t), \\
& \tilde{\mathbf{H}}_{\mathrm{p}}(\mathbf{r}, t)=\int_{V_{\mathrm{p}}} \mathrm{d}^{3} \mathbf{R} \int_{0}^{\infty} \mathrm{d} \omega \frac{\exp (\mathrm{i} k \rho-\mathrm{i} \omega t)}{\rho} \mathbf{H}_{\mathrm{p}}(\mathbf{R}, \hat{\boldsymbol{\rho}}, \omega, t),
\end{aligned}
$$

where $\mathbf{R}$ is the position vector of an infinitesimal element of the volume $V_{\mathrm{p}} ; \boldsymbol{\rho}=\mathbf{r}-\mathbf{R} ; \rho=|\boldsymbol{\rho}| ; \hat{\boldsymbol{\rho}}=\boldsymbol{\rho} / \rho ; \mathbf{E}_{\mathrm{p}}(\mathbf{R}, \hat{\boldsymbol{\rho}}, \omega, t)$ is the complex electric-field amplitude of the quasi-monochromatic spherical wave created by the infinitesimal volume element $d^{3} \mathbf{R}$ in the direction $\hat{\boldsymbol{\rho}}$ at the frequency $\omega$; and

$$
\mathbf{H}_{\mathrm{p}}(\mathbf{R}, \hat{\boldsymbol{\rho}}, \omega, t)=\sqrt{\frac{\epsilon_{0}}{\mu_{0}}} \hat{\boldsymbol{\rho}} \times \mathbf{E}_{\mathrm{p}}(\mathbf{R}, \hat{\boldsymbol{\rho}}, \omega, t) .
$$

Since $k \rho \gg 1$, the integrands in Eqs. (9) and (10) can be thought of as representing locally quasi-plane wavefronts. Again, the electric-field amplitude $\mathbf{E}_{\mathrm{p}}(\mathbf{R}, \hat{\rho}, \omega, t)$ is assumed to vary in time much more slowly than $\exp (-\mathrm{i} \omega t)$, while its quasi-random temporal fluctuations are caused by those of both the amplitude and the phase of the corresponding real electric field vector.

In what follows, we will discuss the practical consequences of the fundamental formulas (1)-(11).

\section{MEASUREMENT OF THE LOCAL POYNTING VECTOR}

A fundamental property of the electromagnetic field is additivity: the electric and magnetic field vectors of a superposition of several fields are equal to vector sums of the respective individual field vectors. Unlike the field itself, the Poynting vector is not additive: the Poynting vector of a superposition of fields is not, in general, equal to the vector sum of the respective partial Poynting vectors. This factor makes the problem of measuring or calculating the right-hand side of Eq. (1) highly nontrivial. Indeed, the total Poynting vector contains the cross terms between the fields of the various different sources, in addition to the sum of the Poynting-vector contributions of the individual sources. These cross terms are the central feature of the nonadditivity.

Equations (5)-(11) show that the total Poynting vector at a point $\mathbf{r}$ is contributed to by locally quasi-plane electromagnetic wavefronts with a wide range of propagation directions from locations both on the star and the planet (Fig. 2). If the solid angle of incoming directions is smaller than $2 \pi$ (as, for example, in the case of point 1 in Fig. 2) then there is hope that the total Poynting vector can be measurable with a carefully designed radiometer. Often, however, the solid angle of incoming directions can exceed $2 \pi$ and pairs of incoming directions can even be opposite to each other, as, for example, in the case of point 2 in Fig. 2 where the waves from the star come from the left and the waves from the planet come generally from the right. Then the Poynting vector cannot be measured with a single radiometer since the instrument will inevitably block certain incoming directions and will thus mismeasure the total local electromagnetic field. Hence we need a simplification of Eqs. (1)-(11) that would enable the measurement of the Poynting vector with two separate radiometers.

Indeed, let us consider a cavity radiometer whose cross section is depicted schematically in Fig. 3. The walls of this radiometer are assumed to be very cold and completely opaque so that the time-averaged Poynting vector at points inside the walls is essentially zero (hereinafter Assumption 1). The entrance window $S_{\text {ent }}$ of the radiometer is flat and normal to the incoming unit vector $\hat{\mathbf{n}}$. Let us consider an imaginary surface $S^{\prime}$ located entirely inside the walls of the radiometer. The union of $S_{\text {ent }}$ and $S^{\prime}$ forms a closed surface $S^{\prime \prime}=S_{\text {ent }} \cup S^{\prime}$ bounding a volume $V^{\prime \prime}$.

Let us consider the situation wherein the radiometer is exposed to a superposition of several quasi-monochromatic plane electromagnetic wavefronts with a wide range of propagation directions $\hat{\mathbf{m}}_{j}$ (Fig. 4). It is obvious that all wavefronts with propagation directions such that $\hat{\mathbf{n}} \cdot \hat{\mathbf{m}}_{j}<0$ (e.g., wavefronts 


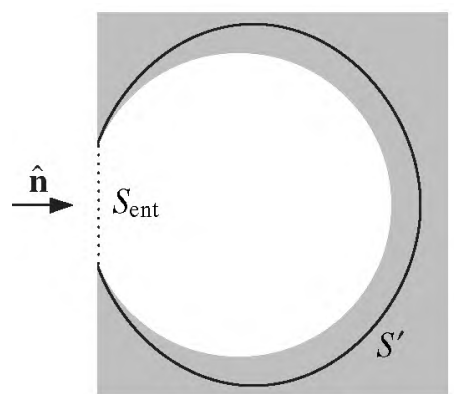

Fig. 3. Hemispherical cavity radiometer.

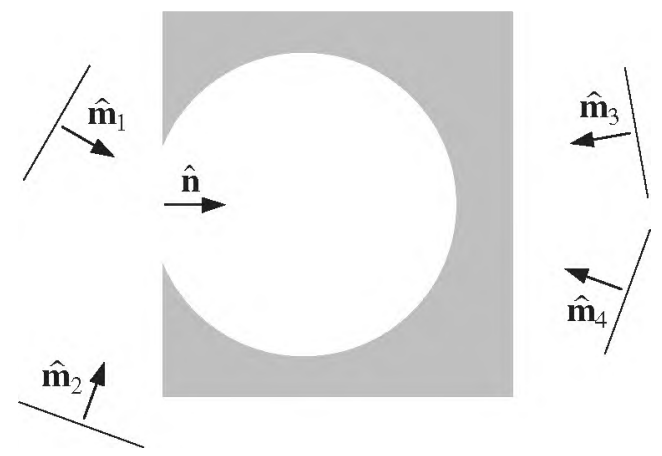

Fig. 4. Hemispherical cavity radiometer exposed to a superposition of quasi-monochromatic plane wavefronts.

3 and 4) will be blocked by the walls of the radiometer and will not contribute to the total electromagnetic field at points $\mathbf{r} \in V^{\prime \prime}$. The superposition of the remaining wavefronts with propagation directions such that $\hat{\mathbf{n}} \cdot \hat{\mathbf{m}}_{j}>0$ (e.g., wavefronts 1 and 2) forms a "truncated" electromagnetic field $\left\{\tilde{\mathbf{E}}_{\mathrm{tr}}(\mathbf{r}, t) ; \tilde{\mathbf{H}}_{\mathrm{tr}}(\mathbf{r}, t)\right\}$ with respect to the original field $\{\tilde{\mathbf{E}}(\mathbf{r}, t) ; \tilde{\mathbf{H}}(\mathbf{r}, t)\}$ of Eqs. (3) and (4).

Let us now assume that the total (i.e., emitted and scattered) electromagnetic field created by the radiometer (including its internal surface) at points $\mathbf{r} \in S_{\text {ent }}$ is negligibly small compared to $\left\{\tilde{\mathbf{E}}_{\mathrm{tr}}(\mathbf{r}, t) ; \tilde{\mathbf{H}}_{\mathrm{tr}}(\mathbf{r}, t)\right\}$ (hereinafter Assumption 2). Then, upon applying the Poynting theorem to the closed surface $S^{\prime \prime}$, we can conclude that the total amount of electromagnetic energy dissipated in the enclosed volume $V^{\prime \prime}$ of the radiometer per unit time is equal to

$$
I_{\mathrm{r}}=\int_{S_{\mathrm{ent}}} \mathrm{d}^{2} \mathbf{r}\left\langle\mathbf{S}_{\mathrm{tr}}(\mathbf{r}, t)\right\rangle \cdot \hat{\mathbf{n}}[\mathrm{W}],
$$

where

$$
\mathbf{S}_{\mathrm{tr}}(\mathbf{r}, t)=\operatorname{Re}\left[\tilde{\mathbf{E}}_{\mathrm{tr}}(\mathbf{r}, t)\right] \times \operatorname{Re}\left[\tilde{\mathbf{H}}_{\mathrm{tr}}(\mathbf{r}, t)\right]\left[\mathrm{Wm}^{-2}\right] .
$$

We will assume that this dissipated power causes warming of the cavity and can thereby be accurately measured (hereinafter Assumption 3).

Equations (12) and (13) provide the first-principles electromagnetic model of what can be called a hemispherical cavity radiometer (HCR). The word "hemispherical" refers to the ability of the instrument to capture the entire hemisphere of

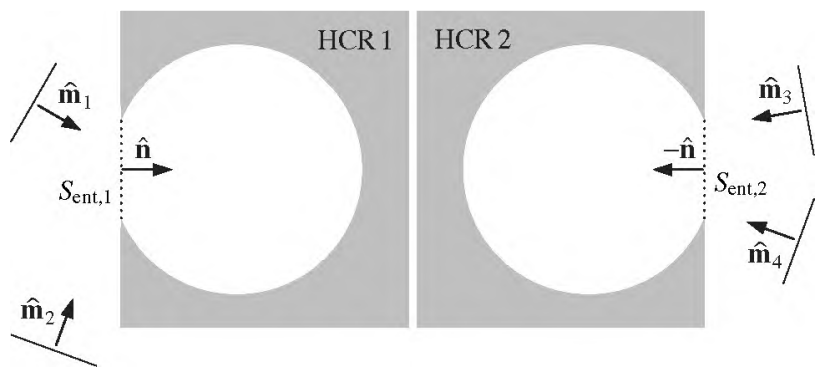

Fig. 5. Combination of two hemispherical cavity radiometers exposed to a superposition of quasi-monochromatic plane wavefronts.

wavefront propagation directions $\hat{\mathbf{m}}$ such that $\hat{\mathbf{m}} \cdot \hat{\mathbf{n}}>0$. In what follows, we will assume that such an instrument can actually be designed and built without discussing any specific technical issues. However, it is imperative to keep in mind the above-formulated Assumptions 1-3 according to which (i) the walls of the radiometer are sufficiently cold, (ii) the surface of the cavity is sufficiently black, and (iii) the electromagnetic energy dissipated inside the volume $V^{\prime \prime}$ per unit time can accurately be measured. Fundamentally, a HCR does not, in general, react to the total Poynting vector at points $\mathbf{r} \in S_{\text {ent }}$ that would exist in the radiometer's absence and instead reacts to the truncated Poynting vector (13).

Let us now consider the measurement configuration involving a combination of two identical but oppositely oriented HCRs 1 and 2 shown in Fig. 5. Obviously, the corresponding truncated electromagnetic field $\left\{\tilde{\mathbf{E}}_{\mathrm{tr}, 1}(\mathbf{r}, t) ; \tilde{\mathbf{H}}_{\mathrm{tr}, 1}(\mathbf{r}, t)\right\}$ is a superposition of waves 1 and 2, while the truncated field $\left\{\mathbf{E}_{\mathrm{tr}, 2}(\mathbf{r}, t) ; \tilde{\mathbf{H}}_{\mathrm{tr}, 2}(\mathbf{r}, t)\right\}$ is a superposition of waves 3 and 4 .

Let us assume that in the absence of the radiometers, the two truncated fields are independent (and thus uncorrelated) random processes at any point $\mathbf{r} \in S_{\text {ent,1 }}$ (hereinafter Assumption 4). This assumption implies that

$$
\langle\mathbf{S}(\mathbf{r}, t)\rangle=\left\langle\mathbf{S}_{\mathrm{tr}, 1}(\mathbf{r}, t)\right\rangle+\left\langle\mathbf{S}_{\mathrm{tr}, 2}(\mathbf{r}, t)\right\rangle, \quad \mathbf{r} \in S_{\mathrm{ent}, 1},
$$

where we have taken into account that

$$
\left\langle\tilde{\mathbf{E}}_{\mathrm{tr}, 1}(\mathbf{r}, t)\right\rangle=\left\langle\tilde{\mathbf{E}}_{\mathrm{tr}, 2}(\mathbf{r}, t)\right\rangle=\left\langle\tilde{\mathbf{H}}_{\mathrm{tr}, 1}(\mathbf{r}, t)\right\rangle=\left\langle\tilde{\mathbf{H}}_{\mathrm{tr}, 2}(\mathbf{r}, t)\right\rangle=0
$$

owing to the high-frequency oscillations of the complex time-harmonic factors involved (see Section 9.3 of [70]).

Note that HCR 1 integrates $\left\langle\mathbf{S}_{\mathrm{tr}, 1}(\mathbf{r}, t)\right\rangle$ over $S_{\text {ent, } 1}$ while HCR 2 integrates $\left\langle\mathbf{S}_{\mathrm{tr}, 2}(\mathbf{r}, t)\right\rangle$ over $S_{\mathrm{ent}, 2}$. However, Eq. (1) actually requires that we integrate both $\left\langle\mathbf{S}_{\mathrm{tr}, 1}(\mathbf{r}, t)\right\rangle$ and $\left\langle\mathbf{S}_{\mathrm{tr}, 2}(\mathbf{r}, t)\right\rangle$ over the same surface. Let us therefore assume that the electric and magnetic field vectors of quasi-monochromatic plane waves 3 and 4 also are independent random processes everywhere in the vicinity of HCRs 1 and 2 (hereinafter Assumption 5). Then it is straightforward to verify that $\left\langle\mathbf{S}_{\mathrm{tr}, 2}(\mathbf{r}, t)\right\rangle$ is independent of $\mathbf{r}$, and is thus the same over $S_{\text {ent } 1}$ and $S_{\text {ent } 2}$. This implies that

$$
\begin{aligned}
\int_{S_{\mathrm{en}, 1}} \mathrm{~d}^{2} \mathbf{r}\left\langle\mathbf{S}_{\mathrm{tr}, 2}(\mathbf{r}, t)\right\rangle \cdot \hat{\mathbf{n}} & =\int_{S_{\mathrm{ent}, 2}} \mathrm{~d}^{2} \mathbf{r}\left\langle\mathbf{S}_{\mathrm{tr}, 2}(\mathbf{r}, t)\right\rangle \cdot \hat{\mathbf{n}} \\
& =-I_{\mathrm{r}, 2}=S_{\mathrm{ent}}\left\langle\mathbf{S}_{\mathrm{tr}, 2}\right\rangle \cdot \hat{\mathbf{n}} .
\end{aligned}
$$


It then becomes clear that

$$
\int_{S_{\mathrm{ens}, 1}} \mathrm{~d}^{2} \mathbf{r}\langle\mathbf{S}(\mathbf{r}, t)\rangle \cdot \hat{\mathbf{n}}=I_{\mathrm{r}, 1}-I_{\mathrm{r}, 2} \cdot
$$

We will refer to the instrument shown in Fig. 5 as the double hemispherical cavity radiometer (DHCR). Equation (17) shows how this instrument can be used to measure the full Poynting vector integrated over the entrance window of its first component (i.e., HCR 1) provided that Assumptions 4 and 5 hold.

Note that, strictly speaking, neither HCR 1 nor HCR 2 in Fig. 5 captures wavefronts propagating perpendicularly to $\hat{\mathbf{n}}$. In what follows, we will assume that such wavefronts represent a set of zero measure with respect to wavefronts with propagation directions $\hat{\mathbf{m}}$ such that $\hat{\mathbf{n}} \cdot \hat{\mathbf{m}} \neq 0$.

\section{MEASUREMENT OF THE PLANETARY ELECTROMAGNETIC-ENERGY BUDGET}

Finally, let us assume that we can fly simultaneously a large number of DHCRs so that they cover the spherical surface $S$ with sufficient density (hereinafter Assumption 6). The optical axes of all the instruments go through the center of the planet and the HCR 1 component of each instrument faces the planet (Fig. 6). In view of Eqs. (1) and (17), we have for the quasi-instantaneous energy budget,

$$
I_{\mathrm{p}}(t) \approx \frac{1}{S_{\text {ent }}} \int_{S} \mathrm{~d}^{2} \mathbf{r}_{0}\left[-I_{\mathrm{r}, 1}\left(\mathbf{r}_{0}, t\right)+I_{\mathrm{r}, 2}\left(\mathbf{r}_{0}, t\right)\right],
$$

where $\mathbf{r}_{0}$ is the position vector of the central point of the entrance window $S_{\text {ent, } 1}$. In practice, only a finite number $N$ of DHCRs can be operational at a moment in time, and so the integral in Eq. (18) has to be replaced by a surface quadrature formula on $S$,

$$
I_{\mathrm{p}}(t) \approx \frac{1}{S_{\text {ent }}} \sum_{n=1}^{N} w_{n}\left[-I_{\mathrm{r}, 1}\left(\mathbf{r}_{0 n}, t\right)+I_{\mathrm{r}, 2}\left(\mathbf{r}_{0 n}, t\right)\right],
$$

where $n$ numbers the instruments and $w_{n}$ are approptiate quadrature weights. Furthermore, typical climate applications

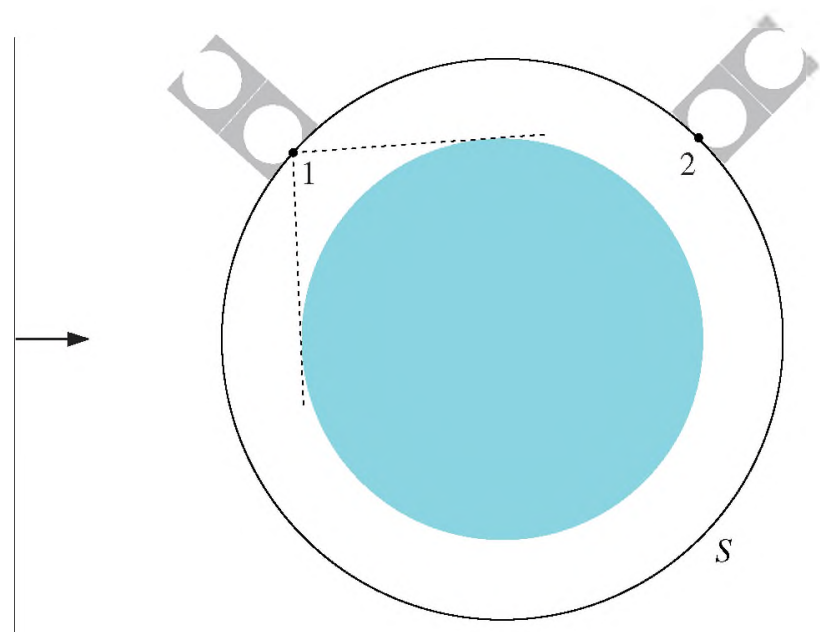

Fig. 6. Measurement of the planetary electromagnetic-energy budget. require averages $\left\langle\left\langle I_{\mathrm{p}}(t)\right\rangle\right\rangle$ over an extended time interval $T$, e.g., over a month, a season, or a year:

$$
\left\langle\left\langle I_{\mathrm{p}}(t)\right\rangle\right\rangle \approx \frac{1}{T} \int_{t-T / 2}^{t+T / 2} \mathrm{~d} t^{\prime} I_{\mathrm{p}}\left(t^{\prime}\right) .
$$

It is clear from the above discussion that $I_{r, 1}(\mathbf{r}, t)$ is contributed to by wavefronts with a large solid angle of incoming directions. This solid angle (shown by dashed lines for point 1 in Fig. 6) typically subtends a vast area of the planetary surface, which implies that $I_{\mathrm{r}, 1}(\mathbf{r}, t)$ cannot be considered representative of specific surface locations. In fact, it is easy to think of situations wherein major contributions to $I_{\mathrm{r}, 1}(\mathbf{r}, t)$ at visible and infrared wavelengths come from widely separated surface elements. For example, the visible-wavelength component of $I_{\mathrm{r}, 1}(\mathbf{r}, t)$ can be overwhelmed by an oblique direction defined by the specular reflection of sunlight from the ocean surface, whereas the infrared component is typically dominated by thermal emission from a nadir surface element.

\section{DISCUSSION AND CONCLUSIONS}

Hansen et al. [28] claim that "Earth's energy imbalance, which must be eliminated to stabilize climate, provides a crucial metric." The entire voluminous body of relevant publications, of which [1-29] are just a representative subset, deals specifically with electromagnetic energy (while often substituting it with the term "radiative energy"), i.e., the energy of the electromagnetic field. One could therefore expect that this particular problem in electromagnetism would have by now been formulated in the framework of an electrodynamics discipline, such as the QED, the semiclassical approach, or classical electromagnetics. Yet, none of the previous publications on this subject have been based on first physical principles, and the term "the Poynting vector" is nowhere to be mentioned. Instead, outdated phenomenological principles and notions are still universally used. This situation is unfortunate given the requisite numerical accuracy as tough as a tenth of a percent or better.

The main advantage of the first-principles framework described in this paper is that it is completely devoid of questionable heuristic and phenomenological concepts. This framework is based on determining the radial component of the local Poynting vector at points of an imaginary spherical surface surrounding the planet, followed by numerical integration over the surface according to Eq. (1). The generality of our approach implies that any first-principles computational scheme or measurement methodology must explicitly be based on Eqs. (1)-(11).

The measurement methodology discussed in Sections 4 and 5 is a natural corollary of the first-principles formalism and invokes only two additional "theoretical" assumptions (Assumptions 4 and 5). Assumption 5 appears to be quite realistic in that the scattering and emission processes in two infinitesimal volume elements of the stellar atmosphere can be expected to be statistically independent unless these volume elements and the observation point lie on the same straight line. Similarly, the wavefronts generated by the star and the planet can also be expected to be statistically independent in the majority of cases, such as that exemplified by point 1 in Fig. 6 . However, Assumption 4 is obviously violated in the case of 
forward scattering of stellar wavefronts by the planetary atmosphere. A well-known manifestation of the forward-scattering coherence is the phenomenon of extinction $[70,71])$. This phenomenon is strongly wavelength-dependent and will manifest itself whenever the instrument captures stellar wavefronts traversing the atmosphere (e.g., point 2 in Fig. 6). Fortunately, however, in such cases HCR 2 captures no wavefronts generated by the star, while HCR 1 automatically captures both the stellar-generated wavefronts and the wavefronts forwardscattered by the planetary atmosphere. These two factors make Assumption 4 unnecessary.

The "instrumental" Assumptions 1-3, as well as the technical capability to achieve and maintain the requisite instrumental calibration, define the feasibility of designing and building an orbital DHCR and are not discussed here. Should one or more of these assumptions prove to be wrong then a different instrument concept will need to be used. In that case a new first-principles measurement methodology must be developed and traced back to Eqs. (1)-(11). It is worth mentioning in this regard that, to the best of our knowledge, the ad hoc approach discussed in $[16,18]$ has never been established as the one based on fundamental principles of classical, semiclassical, and/or quantum electromagnetics.

Assumption 6 has a number of both scientific and logistical aspects. The former have to do with the expected temporal and spatial variability of $I_{\mathrm{r}, 1}\left(\mathbf{r}_{0}, t\right)$ and the length of time intervals used to calculate climate-related averages $\left\langle\left(I_{\mathrm{p}}(t)\right\rangle\right)$. Obviously, flying simultaneously more instruments and/or averaging over longer time intervals can be expected to improve the accuracy of $\left\langle\left\langle I_{\mathrm{p}}(t)\right\rangle\right\rangle$. The logistical aspects have to do with the feasibility of launching and maintaining a constellation of a large number of DHCRs optimally distributed over the surface $S$ as well as with the affordability of this endeavor (cf. [29]). A discussion of these aspects is well beyond the scope of our paper.

Also beyond the scope of this paper is a discussion of whether Eqs. (1)-(11) can be used to develop a theoretical methodology to model the Earth's electromagnetic-energy budget with the requisite accuracy. This problem is extremely complicated, and the applicability of numerical simulations based on the standard phenomenological radiative transfer theory (especially on its scalar version $[72,73]$ ) remains questionable.

Funding. National Aeronautics and Space Administration (NASA).

Acknowledgment. We appreciate numerous insightful discussions with James Hansen and William Rossow and thank two anonymous reviewers for their helpful comments.

\section{REFERENCES}

1. J. Tyndall, Contributions to Molecular Physics in the Domain of Radiant Heat (Appleton and Company, 1873).

2. K. Y. Kondratyev, Radiation in the Atmosphere (Academic, 1969).

3. R. M. Goody and Y. L. Yung, Atmospheric Radiation: Theoretical Basis (Oxford University, 1989).

4. K. N. Liou, Radiation and Cloud Processes in the Atmosphere: Theory, Observation, and Modeling (Oxford University, 1992).

5. K. E. Trenberth, ed., Climate System Modeling (Cambridge University, 1993).
6. D. L. Hartmann, Global Physical Climatology (Academic, 1994).

7. G. E. Thomas and K. Stamnes, Radiative Transfer in the Atmosphere and Ocean (Cambridge University, 1999).

8. D. A. Randall, ed., General Circulation Model Development (Academic, 2000).

9. K. N. Liou, An Introduction to Atmospheric Radiation (Academic, 2002).

10. M. L. Salby, Physics of the Atmosphere and Climate (Cambridge University, 2012)

11. M. Wendisch and P. Yang, Theory of Atmospheric Radiative Transfer: A Comprehensive Introduction (Wiley, 2012).

12. S. J. Mackwell, A. A. Simon-Miller, J. W. Harder, and M. A. Bullock, eds., Comparative Climatology of the Terrestrial Planets (University of Arizona, 2013).

13. M. A. Box and G. P. Box, Physics of Radiation and Climate (CRC Press, 2016).

14. T. H. Vonder Haar and V. E. Suomi, "Measurements of the earth's radiation budget from satellites during a five-year period. Part I: Extended time and space means," J. Atmos. Sci. 28, 305-314 (1971).

15. E. Raschke, T. H. Vonder Haar, W. R. Bandeen, and M. Pasternak, "The annual radiation balance of the Earth-atmosphere system during 1969-1970 from Nimbus-3 measurements," J. Atmos. Sci. 30, 341-364 (1973).

16. B. R. Barkstrom and G. L. Smith, "The Earth radiation budget experiment: science and implementation," Rev. Geophys. 24, 379-390 (1986)

17. S. Q. Kidder and T. H. Vonder Haar, Satellite Meteorology: An Introduction (Academic, 1995).

18. B. A. Wielicki, B. R. Barkstrom, E. F. Harrison, R. B. Lee III, G. L. Smith, and J. E. Cooper, "Clouds and the Earth's Radiant Energy System (CERES): an Earth observing system experiment," Bull. Am. Meteorol. Soc. 77, 853-868 (1996).

19. J. Hansen, M. Sato, P. Kharecha, and K. Von Schuckmann, "Earth's energy imbalance and implications," Atmos. Chem. Phys. 11, 13421-13449 (2011).

20. R. Kandel, "Understanding and measuring Earth's energy budget: from Fourier, Humboldt, and Tyndall to CERES and beyond," Surv. Geophys. 33, 337-350 (2012).

21. M. D. Palmer, "Climate and Earth's energy flows," Surv. Geophys. 33, 351-357 (2012).

22. N. G. Loeb, S. Kato, W. Su, T. Wong, F. G. Rose, D. R. Doelling, J. R. Norris, and X. Huang, "Advances in understanding top-of-atmosphere radiation variability from satellite observations," Surv. Geophys. 33, 359-385 (2012).

23. K. E. Trenberth and J. T. Fasullo, "Tracking Earth's energy: from El Nin̄o to global warming," Surv. Geophys. 33, 413-426 (2012).

24. B. Stevens and S. E. Schwartz, "Observing and modeling Earth's energy flows," Surv. Geophys. 33, 779-816 (2012).

25. G. L. Stephens, J. Li, M. Wild, C. A. Clayson, N. Loeb, S. Kato, T. L'Ecuyer, and P. W. Stackhouse, Jr., M. Lebsock and T. Andrews, "An update on Earth's energy balance in light of the latest global observations," Nat. Geosci. 5, 691-696 (2012).

26. K. E. Trenberth, Y. Zhang, J. T. Fasullo, and S. Taguchi, "Climate variability and relationships between top-of-atmosphere radiation and temperatures on Earth," J. Geophys. Res. Atmos. 120, 36423659 (2015).

27. G. L. Stephens, D. O'Brien, P. J. Webster, P. Pilewski, S. Kato, and J.-L. Li, "The albedo of Earth," Rev. Geophys. 53, 141-163 (2015).

28. J. Hansen, M. Sato, P. Hearty, R. Ruedy, M. Kelley, V. MassonDelmotte, G. Russell, G. Tselioudis, J. Cao, E. Rignot, I. Velicogna, E. Kandiano, K. Von Schuckmann, P. Kharecha, A. N. Legrande, M. Bauer, and K.-W. Lo, "Ice melt, sea level rise and superstorms: evidence from paleoclimate data, climate modeling, and modern observations that $2^{\circ} \mathrm{C}$ global warming is highly dangerous," Atmos. Chem. Phys. Discuss. 15, 20059-20179 (2015).

29. W. H. Swartz, L. P. Dyrud, S. R. Lorentz, D. L. Wu, W. J. Wiscombe, S. J. Papadakis, P. M. Huang, E. L. Reynolds, A. W. Smith, and D. M. Deglaua, "The RAVAN CubeSat mission: advancing technologies for climate observation," in Geoscience and Remote Sensing Symposium (IGARSS) (IEEE, 2015), pp. 5300-5303. 
30. P. Bouguer, Traité d'Optique sur la Gradation de la Lumiere (Académie Royale des Sciences, 1760).

31. J. H. Lambert, Photometria, sive de Mensura et Gradibus Luminis, Colorum et Umbrae (Detlefsen, 1760).

32. A. Beer, Grundriss des Photometrischen Calcüles (Friedrich Vieweg und Sohn, 1854).

33. E. Lommel, "Die Photometrie der diffusen Zurückwerfung," Sitzber. Acad. Wissensch. München 17, 95-124 (1887).

34. O. Chwolson, "Grundzüge einer mathematischen Theorie der inneren Diffusion des Lichtes," Bull. l'Acad. Impériale Sci. St. Pétersbourg 33, 221-256 (1889).

35. A. Schuster, "Radiation through a foggy atmosphere," Astrophys. J. 21, 1-22 (1905).

36. M. Planck, Theorie der Wärmestrahlung (Verlag Von Johann Ambrosius Barth, 1906).

37. A. A. Gershun, "The light field," J. Phys. Math. 18, 51-151 (1939).

38. S. Chandrasekhar, Radiative Transfer (Oxford University, 1950).

39. J. C. Maxwell, A Treatise on Electricity and Magnetism (Clarendon, 1873).

40. E. Wolf, "Coherence and radiometry," J. Opt. Soc. Am. 68, 6-17 (1978).

41. R. Kidd, J. Ardini, and A. Anton, "Evolution of the modern photon," Am. J. Phys. 57, 27-35 (1989).

42. W. E. Lamb, Jr., "Anti-photon," Appl. Phys. B 60, 77-84 (1995).

43. L. Mandel and E. Wolf, Quantum Coherence and Quantum Optics (Cambridge University, 1995).

44. L. A. Apresyan and Yu. A. Kravtsov, Radiation Transfer: Statistical and Wave Aspects (Gordon and Breach, 1996).

45. M. I. Mishchenko, "Gustav Mie and the fundamental concept of electromagnetic scattering by particles: a perspective," J. Quant. Spectrosc. Radiat. Transfer 110, 1210-1222 (2009).

46. M. I. Mishchenko, "Directional radiometry and radiative transfer: the convoluted path from centuries-old phenomenology to physical optics," J. Quant. Spectrosc. Radiat. Transfer 146, 4-33 (2014).

47. C. Cohen-Tannoudij, J. Dupont-Roc, and G. Grynberg, Photons and Atoms: Introduction to Quantum Electrodynamics (Wiley, 1989).

48. C. Cohen-Tannoudij, J. Dupont-Roc, and G. Grynberg, Atom-Photon Interactions: Basic Processes and Applications (Wiley, 1992).

49. J. J. Sakurai, Advanced Quantum Mechanics (Addison-Wesley, 1980).

50. J. N. Dodd, Atoms and Light: Interactions (Plenum, 1991).

51. G. Grynberg, A. Aspect, and C. Fabre, Introduction to Quantum Optics: From the Semi-classical Approach to Quantized Light (Cambridge University, 2010).

52. L. D. Landau and E. M. Lifshitz, The Classical Theory of Fields (Butterworth-Heinemann, 1994).
53. K. E. Oughstun, Electromagnetic and Optical Pulse Propagation 1 (Springer, 2006)

54. J. H. Poynting, "On the transfer of energy in the electromagnetic field," Phil. Trans. R. Soc. London 175, 343-361 (1884).

55. O. Heaviside, "Electromagnetic induction and its propagation," The Electrician 14, 178-180; 306-307 (1885).

56. H. A. Lorentz, The Theory of Electrons (B. G. Teubner, 1916).

57. D. Bohm, Quantum Theory (Prentice-Hall, 1951).

58. H. A. Kramers, Quantum Mechanics (North-Holland, 1957).

59. J. J. Duderstadt and W. R. Martin, Transport Theory (Wiley, 1979).

60. G. I. Marchuk, G. A. Mikhailov, M. A. Nazaraliev, R. A. Darbinjan, B. A Kargin, and B. S. Elepov, The Monte Carlo Methods in Atmospheric Optics (Springer, 1980).

61. J. Lenoble, ed., Radiative Transfer in Scattering and Absorbing Atmospheres: Standard Computational Procedures (A. Deepak, 1985).

62. W. J. Wiscombe, "Scales, tools and reminiscences," in 3D Radiative Transfer in Cloudy Atmospheres, A. Marshak and A. B. Davis, eds. (Springer, 2005), pp. 3-92.

63. C. F. Bohren and E. E. Clothiaux, Fundamentals of Atmospheric Radiation (Wiley, 2006)

64. W. Zdunkowski, T. Trautmann, and A. Bott, Radiation in the Atmosphere (Cambridge University, 2007).

65. A. J. Welch and M. J. C. van Gemert, eds., Optical-Thermal Response of Laser-Irradiated Tissue (Springer, 2011).

66. O. Pujol, "Comment on the (misused) concept of photon in radiative transfer, and proposition of a neologism," J. Quant. Spectrosc. Radiat. Transfer 159, 29-31 (2015).

67. I. Hubeny and D. Mihalas, Theory of Stellar Atmospheres (Princeton University, 2015).

68. C. Brosseau, Fundamentals of Polarized Light: A Statistical Optics Approach (Wiley, 1998).

69. A. Lipson, S. G. Lipson, and H. Lipson, Optical Physics (Cambridge University, 2011).

70. M. I. Mishchenko, Electromagnetic Scattering by Particles and Particle Groups: An Introduction (Cambridge University, 2014).

71. M. I. Mishchenko, L. D. Travis, and A. A. Lacis, Multiple Scattering of Light by Particles: Radiative Transfer and Coherent Backscattering (Cambridge University, 2006).

72. M. I. Mishchenko, A. A. Lacis, and L. D. Travis, "Errors induced by the neglect of polarization in radiance calculations for Rayleigh-scattering atmospheres," J. Quant. Spectrosc. Radiat. Transfer 51, 491-510 (1994).

73. A. A. Lacis, J. Chowdhary, M. I. Mishchenko, and B. Cairns, "Modeling errors in diffuse-sky radiation: vector vs. scalar treatment," Geophys. Res. Lett. 25, 135-138 (1998). 